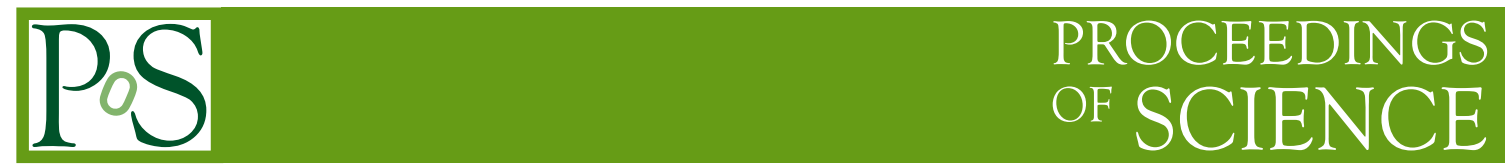

\title{
KN scattering with hadrons exchange potential in 3D technique
}

\author{
Agus Salam* \\ Universitas Indonesia \\ E-mail: agussalamefisika.ui.ac.id \\ Imam Fachruddin \\ Universitas Indonesia
}

We have formulated the KN scattering in a three-dimensional (3D) technique. The LippmannSchwinger equation is evaluated in 3D basis states without partial-wave (PW) expansion. The result is a set of two coupled integral equations for T-matrix elements in two variables, namely the momentum magnitude and the scattering angle. For the KN interaction we employ an already derived hadrons exchange model with the second order contributions only. The parameters of the model will be later determined by fitting to the experimental data for the cross section and some spin observables.

XV International Conference on Hadron Spectroscopy 4-8/11/2013

Nara, Japan

\footnotetext{
* Speaker.
} 


\section{Introduction}

The partial-wave (PW) expansion has been a successful technique in solving few-particle systems, see for instance Ref. [1]. But as the energy increases PW calculations become more tedious both algebraically and numerically, since many more higher angular momentum states have to be considered. The three-dimensional (3D) technique appears to be a good alternative to the PW one in solving the Lippmann-Schwinger (LS) equation, see for example Ref. [2,3]. In 3D technique the linear momentum state as part of the basis state is not expanded in the angular momentum states, thus, all partial waves are taken.

In Ref. [4] we compare PW calculations to 3D calculations for the cross section of KN scattering based on a simple Yukawa-tipe interaction with a spin-orbit force. We find that for higher energies of a few hundreds $\mathrm{MeV}$, the PW calculations converge slowly. This suggests us more to use the 3D technique especially for higher energies. In this work we step further in our investigation on $\mathrm{KN}$ scattering using the 3D technique by taking a more realistic $\mathrm{KN}$ interaction derived in Ref. [5]. Our future plan is to refit the parameters of the potential by comparing our 3D calculations to experimental data for the cross section and some spin observables.

We describe the KN interaction that we take in Section 2. In Section 3 we show the 3D formulation. We summarize in Section 4.

\section{Hadron Exchange Interaction}

Here we describe briefly the KN interaction model developed by Büttgen et al. [5]. We show only those part of the model that we take in our present work. We take only up to the second order in accordance with our attemp to create a kind of one-hadron-exchange potential for $\mathrm{KN}$ system. The $\mathrm{KN}$ interaction model in Ref. [5] considers mesons and baryons as the degrees of freedom of the strong interaction dynamics. In this approach, the interactions are represented by the meson-mesonmeson and baryon-baryon-meson vertices. For the baryon-baryon-scalar meson (bbs), baryonbaryon-pseudoscalar meson ( $b b p$ ), baryon-baryon-vector meson ( $b b v)$, pseudoscalar-pseudoscalarscalar meson ( $p p s)$, and pseudoscalar-pseudoscalar-vector meson ( $p p v$ ) vertices, respectively, the interaction Lagrangians take the following forms

$$
\begin{aligned}
& \mathscr{L}_{b b s}=g_{b b s} \bar{\Psi}_{b} \Psi_{b} \Phi_{s} \\
& \mathscr{L}_{b b p}=g_{b b p} \bar{\Psi}_{b} i \gamma_{5} \Psi_{b} \Phi_{p} \\
& \mathscr{L}_{b b v}=g_{b b v} \bar{\Psi}_{b} \gamma_{\mu} \Psi_{b} \Phi_{v}^{\mu}+\frac{f_{b b v}}{4 m_{N}} \bar{\Psi}_{b} \sigma_{\mu v} \Psi_{b}\left(\partial^{\mu} \Phi_{v}^{v}-\partial^{v} \Phi_{v}^{\mu}\right) \\
& \mathscr{L}_{p p s}=g_{p p s} m_{p} \Phi_{p} \Phi_{p} \Phi_{s} \\
& \mathscr{L}_{p p v}=g_{p p v} \Phi_{p}\left(\partial_{\mu} \Phi_{p}\right) \Phi_{v}^{\mu} .
\end{aligned}
$$

Here $\Psi_{b}$ is the baryon field operator and $\Phi_{s}, \Phi_{p}$, and $\Phi_{v}^{\mu}$ are the field operators for scalar, pseudoscalar, and vector mesons, respectively. Note that in the expressions above only the space-spin part is given. The isospin factor will be inserted in the equation describing the potential (see explanations below). As explained in Ref. [5] only the $\sigma$-meson is considered for the scalar meson, $K$-meson for the pseudoscalar meson, $\rho$ - and $\omega$-meson for the vector mesons, and the nucleon $N$, $\Lambda$-, and $\Sigma$-hyperon for the baryons. The second-order time-ordered diagrams are shown in Fig. 1. 


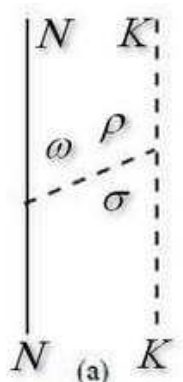

(a)

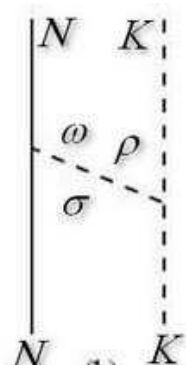

(b)

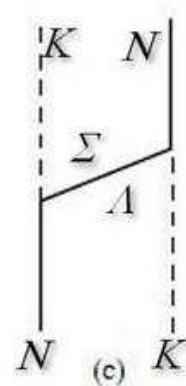

(c)

Figure 1: Time ordered diagrams for hadrons exchange interaction that is used in this work.

The diagrams in Fig. 1 lead to the following hadron exchange interactions [5]. The $\sigma$-meson exchange (diagrams (a)+(b)) gives

$$
\left\langle\vec{p}^{\prime} \lambda^{\prime}\left|V_{\sigma}\right| \vec{p} \lambda\right\rangle=\frac{g_{K K \sigma} g_{N N \sigma}}{16 \pi^{3}} \frac{F_{K K \sigma} F_{N N \sigma}}{\sqrt{\omega_{K} \omega_{K}^{\prime}}} \frac{\bar{u}\left(\vec{p}^{\prime} \lambda^{\prime}\right) u(\vec{p} \lambda)}{q_{\sigma}^{2}-m_{\sigma}^{2}} .
$$

The vector meson $(v=\rho, \omega)$ exchange (diagrams (a)+(b)) gives

$$
\begin{aligned}
\left\langle\vec{p}^{\prime} \lambda^{\prime}\left|V_{v}\right| \vec{p} \lambda\right\rangle= & -\frac{g_{K K v}}{32 \pi^{3}} \frac{F_{K K v} F_{N N v}}{\sqrt{\omega_{K} \omega_{K}^{\prime}}} \frac{1}{q_{v}^{2}-m_{v}^{2}}\left(\left(g_{N N v}+f_{N N v}\right)\left(p_{K}^{\prime}+p_{K}\right)^{\mu} \bar{u}\left(\vec{p}^{\prime} \lambda^{\prime}\right) \gamma_{\mu} u(\vec{p} \lambda)\right. \\
& \left.-\frac{f_{N N v}}{2 m_{N}}\left(p_{N}+p_{N}^{\prime}\right)^{\mu}\left(p_{K}+p_{K}^{\prime}\right)_{\mu} \bar{u}\left(\vec{p}^{\prime} \lambda^{\prime}\right) u(\vec{p} \lambda)\right) .
\end{aligned}
$$

Since the $\rho$-meson has isospin 1, the corresponding contribution should be multiplied by an isospin factor $\tau_{1} \cdot \tau_{2}$. Note that both kaon and nucleon have isospin $\frac{1}{2}$. The hyperon $(Y=\Lambda, \Sigma)$ exchange (diagram (c)) gives

$$
\left\langle\vec{p}^{\prime} \lambda^{\prime}\left|V_{Y}\right| \vec{p} \lambda\right\rangle=\frac{g_{K Y N}^{2}}{32 \pi^{3}} \frac{F_{K Y N}^{2}}{\sqrt{\omega_{K} \omega_{K}^{\prime}}} \frac{\bar{u}\left(\vec{p}^{\prime} \lambda^{\prime}\right)\left(\gamma_{\mu} p_{Y}^{\mu}-m_{Y}\right) u(\vec{p} \lambda)}{E_{Y}\left(z-\omega_{K}^{\prime}-\omega_{K}+E_{Y}+i \varepsilon\right)}
$$

with $z=E_{N}+\omega_{K}$. The isospin factor for $\Lambda$-exchange is $\frac{1}{2}\left(1+\tau_{1} \cdot \tau_{2}\right)$ and for $\Sigma$-exchange is $\frac{1}{2}(3-$ $\left.\tau_{1} \cdot \tau_{2}\right)$. In these formulations, $\lambda$ is the quantum number of the spin projection in some prefered direction and $\vec{p}$ and $\vec{p}^{\prime}$ are the relative momentum of kaon-nucleon system in the initial and final states, respectively. In order to consider the hadron structure, Ref. [5] applies the form factor

$$
F_{h}=\left(\frac{\Lambda_{h}^{2}-m_{h}^{2}}{\Lambda_{h}^{2}+\vec{q}_{h}^{2}}\right)^{n_{h}}
$$

for meson-meson-meson vertices with $n_{h}=1(h=\sigma, \omega, \rho)$ and $\vec{q}_{h}=\vec{p}^{\prime}-\vec{p}$ is the transfered momentum. For hyperon containing vertices, Ref. [5] applies the form factor

$$
F_{Y}=\left(\frac{\Lambda_{Y}^{4}+m_{Y}^{4}}{\Lambda_{Y}^{4}+q_{Y}^{4}}\right)
$$

with $q_{Y}$ is the transfered 4-momentum. As mentioned in Ref. [5], the reason for taking this form factors is to consider the off-shell behaviour of a hyperon exchanged. 


\section{Three-dimensional (3D) Formulation}

The basis state in the 3D technique is defined as [4]

$$
|\vec{p} \lambda\rangle \equiv|\vec{p}\rangle|\hat{z} \lambda\rangle
$$

which is a direct product of the linear momentum state $|\vec{p}\rangle$ dan the spin state $|\hat{z} \lambda\rangle$ with the spin being quantized along the $z$-axis and $\lambda= \pm \frac{1}{2}$. In this basis, the LS equation is obtained as

$$
T_{\lambda^{\prime} \lambda}\left(\vec{p}^{\prime}, \vec{p}\right)=V_{\lambda^{\prime} \lambda}\left(\vec{p}^{\prime}, \vec{p}\right)+\sum_{\lambda^{\prime \prime}} \int d \vec{p}^{\prime \prime} V_{\lambda^{\prime} \lambda^{\prime \prime}}\left(\vec{p}^{\prime}, \vec{p}^{\prime \prime}\right) G_{0}^{+}\left(E_{p^{\prime \prime}}, E_{p}\right) T_{\lambda^{\prime \prime} \lambda}\left(\vec{p}^{\prime \prime}, \vec{p}\right),
$$

where

$$
\begin{aligned}
T_{\lambda^{\prime} \lambda}\left(\vec{p}^{\prime}, \vec{p}\right) & \equiv\left\langle\vec{p}^{\prime} \lambda^{\prime}|T| \vec{p} \lambda\right\rangle \\
V_{\lambda^{\prime} \lambda}\left(\vec{p}^{\prime}, \vec{p}\right) & \equiv\left\langle\vec{p}^{\prime} \lambda^{\prime}|V| \vec{p} \lambda\right\rangle=\sum_{h}\left\langle\vec{p}^{\prime} \lambda^{\prime}\left|V_{h}\right| \vec{p} \lambda\right\rangle
\end{aligned}
$$

with $h=\sigma, \rho, \omega, \Lambda, \Sigma$. The free propagator is given as

$$
G_{0}^{+}\left(E_{p^{\prime \prime}}, E_{p}\right)=\lim _{\varepsilon \rightarrow 0} \frac{1}{E_{p}+i \varepsilon-E_{p^{\prime \prime}}}
$$

where $E_{p}=p^{2} / 2 \mu$ is the initial kinetic energy of the system, which is also the energy at which the scattering occurs.

The $\mathrm{KN}$ potential in momentum space has a general structure as

$$
\begin{aligned}
V_{h}\left(\vec{p}^{\prime}, \vec{p}\right) & =\left\langle\vec{p}^{\prime}\left|V_{h}\right| \vec{p}\right\rangle \\
& =A_{h}\left(\vec{p}^{\prime}, \vec{p}\right)+B_{h}\left(\vec{p}^{\prime}, \vec{p}\right) \vec{\sigma} \cdot \vec{p}^{\prime} \vec{\sigma} \cdot \vec{p} .
\end{aligned}
$$

with $A_{h}\left(\vec{p}^{\prime}, \vec{p}\right)$ and $B_{h}\left(\vec{p}^{\prime}, \vec{p}\right)$ being some spin-independent function and $\vec{\sigma}$ are the Pauli's matrices. We evaluate the matrix element of the spin operator in the last term of Eq.(3.6) and obtain

$$
\begin{aligned}
\left\langle\hat{z} \lambda^{\prime}\left|\vec{\sigma} \cdot \vec{p}^{\prime} \vec{\sigma} \cdot \vec{p}\right| \hat{z} \lambda\right\rangle= & \delta_{\lambda^{\prime} \lambda}\left(\cos \theta^{\prime} \cos \theta+e^{-2 i \lambda\left(\phi^{\prime}-\phi\right)} \sin \theta^{\prime} \sin \theta\right) \\
& +\delta_{\lambda^{\prime},-\lambda} 2 \lambda e^{2 i \lambda \phi^{\prime}}\left(\sin \theta^{\prime} \cos \theta-e^{-2 i \lambda\left(\phi^{\prime}-\phi\right)} \cos \theta^{\prime} \sin \theta\right) .
\end{aligned}
$$

In the case $\vec{p}=p \hat{z}$, the azimutal behaviour of $V_{\lambda^{\prime} \lambda}\left(\vec{p}^{\prime}, \vec{p}\right)$ and $T_{\lambda^{\prime} \lambda}\left(\vec{p}^{\prime}, \vec{p}\right)$ show up as

$$
\begin{aligned}
& V_{\lambda^{\prime} \lambda}\left(\vec{p}^{\prime}, p \hat{z}\right)=e^{-i\left(\lambda^{\prime}-\lambda\right) \phi^{\prime}} V_{\lambda^{\prime} \lambda}\left(p^{\prime}, p, \theta^{\prime}\right) \\
& T_{\lambda^{\prime} \lambda}\left(\vec{p}^{\prime}, p \hat{z}\right)=e^{-i\left(\lambda^{\prime}-\lambda\right) \phi^{\prime}} T_{\lambda^{\prime} \lambda}\left(p^{\prime}, p, \theta^{\prime}\right) .
\end{aligned}
$$

This azimuthal behaviour leads to the final form of the integral equation for the $T$-matrix element

$$
\begin{aligned}
T_{\lambda^{\prime} \lambda}\left(p^{\prime}, p^{\prime} \theta^{\prime}\right)= & V_{\lambda^{\prime} \lambda}\left(p^{\prime}, p^{\prime} \theta^{\prime}\right)+2 \mu \lim _{\varepsilon \rightarrow \infty} \sum_{\lambda^{\prime \prime}} \int_{0}^{\infty} d p^{\prime \prime} \frac{p^{\prime \prime 2}}{p^{2}+i \varepsilon-p^{\prime 2}} \\
& \times \int_{-1}^{1} d \cos \theta^{\prime \prime} V_{\lambda^{\prime} \lambda^{\prime \prime}}^{\lambda}\left(p^{\prime}, p^{\prime \prime}, \theta^{\prime}, \theta^{\prime \prime}\right) T_{\lambda^{\prime \prime} \lambda}\left(p^{\prime \prime}, p^{\prime} \theta^{\prime \prime}\right)
\end{aligned}
$$


with

$$
V_{\lambda^{\prime} \lambda^{\prime \prime}}^{\lambda}\left(p^{\prime}, p^{\prime \prime}, \theta^{\prime}, \theta^{\prime \prime}\right)=\int_{0}^{2 \pi} d \phi^{\prime \prime} V_{\lambda^{\prime} \lambda^{\prime \prime}}\left(\vec{p}^{\prime}, \vec{p}^{\prime \prime}\right) e^{i\left(\lambda^{\prime} \phi^{\prime}-\lambda^{\prime \prime} \phi^{\prime \prime}\right)} e^{-i \lambda\left(\phi^{\prime}-\phi^{\prime \prime}\right)} .
$$

The symmetry behaviour of $T_{\lambda^{\prime} \lambda}\left(p^{\prime}, p, \theta^{\prime}\right)$ shows up as [4]

$$
T_{\lambda^{\prime} \lambda}\left(p^{\prime}, p, \theta^{\prime}\right)=(-)^{\lambda^{\prime}-\lambda} T_{-\lambda^{\prime},-\lambda}\left(p^{\prime}, p, \theta^{\prime}\right),
$$

thus, allowing us to solve Eq.(3.10) only for $T_{\frac{1}{2} \frac{1}{2}}\left(p^{\prime}, p, \theta^{\prime}\right)$ and $T_{-\frac{1}{2} \frac{1}{2}}\left(p^{\prime}, p^{\prime} \theta^{\prime}\right)$.

Having the $T$-matrix element $T_{\frac{1}{2} \frac{1}{2}}\left(p^{\prime}, p, \theta^{\prime}\right)$ and $T_{-\frac{1}{2} \frac{1}{2}}\left(p^{\prime}, p^{\prime} \theta^{\prime}\right)$, we can calculate the spinaveraged differential cross section as

$$
I_{0} \equiv \frac{d \sigma}{d \Omega}=\left(4 \pi^{2} \mu\right)^{2}\left(\left|T_{\frac{1}{2} \frac{1}{2}}\left(p, p, \theta^{\prime}\right)\right|^{2}+\left|T_{-\frac{1}{2} \frac{1}{2}}\left(p, p, \theta^{\prime}\right)\right|^{2}\right)
$$

and the polarization, as one example of the spin observable, as

$$
P_{y}=\frac{2}{I_{0}}\left(4 \pi^{2} \mu\right)^{2} \operatorname{Im}\left(T_{\frac{1}{2} \frac{1}{2}}^{*}\left(p, p, \theta^{\prime}\right) T_{-\frac{1}{2} \frac{1}{2}}\left(p, p, \theta^{\prime}\right)\right) .
$$

\section{Summary}

The KN scattering has been formulated by using a 3D technique. The hadrons exchange model has been applied for the KN interaction by considering the second order diagrams only. The cross section and some spin observables will be utilized in determining the parameters of the model. All this is needed for our intention to make a one-hadron-exchange $\mathrm{KN}$ interaction in the next future.

\section{Acknowledgement}

This work was supported in part by the University of Indonesia under the BOPTN 2013 Grant.

\section{References}

[1] Glöckle, W., et al.: The three nucleon continuum: achievements, challenges, and applications. Phys. Rep.274, 107 (1996).

[2] Elster, Ch., Thomas, J.H., Glöckle, W.: Two-body T matrices without angular momentum decomposition: Energy and momentum dependencies. Few-Body Systems 24, 55 (1998)

[3] Fachruddin, I., Salam, A.: Scattering of a spin-1/2 particle off a spin-0 target in a simple three-dimensional basis. Few-Body System 54, 221 (2013). DOI: 10.1007/s00601-012-0353-y

[4] Salam, A., Fachruddin, I.: KN Scattering in 3D Formulation. Few-Body System 54, 1625 (2013). DOI 10.1007/s00601-012-0557-1

[5] Büttgen, R., et al.: A Meson Exchange Model For The K+ N Interaction. Nucl. Phys. A 506, 586 (1990) 